\title{
Ultrasonic Scattering Attenuation in Nodular Cast Iron: Experimental and Simulation Studies
}

\author{
Mário Santos* - Jaime Santos \\ University of Coimbra, CEMMPRE, Department of Electrical and Computer Engineering, Portugal
}

This work evaluates the ultrasonic scattering attenuation of structures with complex scatterer distributions via experimental and simulation studies. The proposed approach uses experimental attenuation knowledge to infer the scatterer size and its concentration in the studied structures, which are important for the effective construction of simulated models. The MATLAB $k$-Wave toolbox has been used to implement the simulator. Several cast-iron samples have been used to demonstrate the importance of simulation in the characterization of such structures. First, the scattering attenuation was evaluated using the Truell and Papadakis models, and then the results were compared with experimental ones. Emphasis was given to the Papadakis approach because it takes into account the scatterer size distribution. It is demonstrated that both analytical models provide results that are far from the experimental ones. The developed simulator for the studied samples led to a predictive model, in which the attenuation was proportional to the fifth power of the scatterer size, and the corresponding formulation is close to the one proposed by the analytical models.

Keywords: modelling, anisotropy, pulse-echo, simulation, ultrasonic attenuation

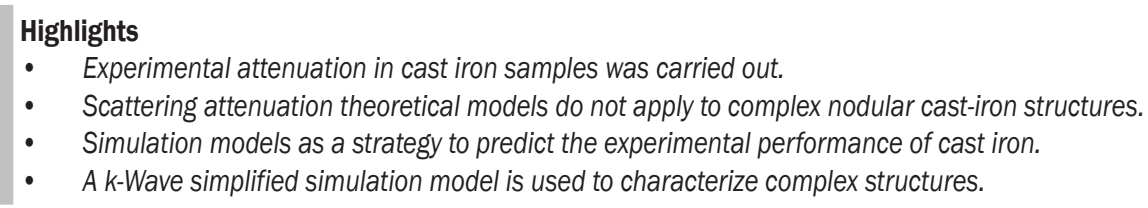

\section{O INTRODUCTION}

There are many applications for nodular cast iron due to its castability, high thermal conductivity, and good mechanical properties, specifically tensile strength and ductility. The mechanical properties of a metal greatly depend on the microstructure; in the case of nodular cast iron, which is produced by adding, shortly before solidification, a small amount (lower than 0.04 $\%)$ of substances such as magnesium or cerium are present. These substances give rise to the growth of nodular graphite, whose shape and distribution are of fundamental importance in the behaviour of the metal [1] to [3]. Thus, the non-destructive evaluation of such structures is very important for the identification of the nodularity and matrix phases.

Ultrasonic characterization offers great advantages when compared with destructive metallographic methods. The interaction of ultrasound waves with the material microstructure can be evaluated, measuring the acoustic parameters, including velocity and attenuation. Two ultrasound attenuation mechanisms are generally identified: absorption and scattering. Absorption is related to thermal conduction loss, hysteresis, and a viscous loss mechanism [4]. Scattering is due to heterogeneities such as grain boundaries, voids, inclusions, secondphase particles or porosity [5] to [9]. This attenuation mechanism is commonly accepted as the most important in heterogeneous materials, such as the case of cast iron [10] to [13]. It is important to take into account the fact that the scattering effects of the matrix grains are too small when compared to the nodular scattering effects and can be ignored [6], [7], [14] to [16].

Several authors have extensively studied scattering attenuation. At the beginning of the last century, Rayleigh presented a scattering formula [17], later adapted by Mason and McSkimin [18] and [19]; the case of polycrystalline aluminium. Huntington [20] used a stochastic theory to explain the scattering effects in polycrystalline structures. Lifshitz and Parkhomovskii [21] proposed a theory that considers the mode conversion at the grain boundaries. Moreover, a great contribution was made by Papadakis [5], [6], [22] to [25] with several published works related to that topic. The author classified the scattering in three classical regimes depending on the relation between the grain size (D) and the wavelength $(\lambda)$ : (1) Rayleigh regime (for $\lambda>>D$ ), where the attenuation is proportional to the fourth power of frequency; (2) stochastic regime (for $\lambda \cong D$ ), where the attenuation is proportional to the square of frequency; (3) and geometrical regime (for $\lambda<<D$ ), where the attenuation is frequency independent. For each regime, the microstructure is assumed to be 
composed of spherical grains of the same size filling the medium. As the Mason and McSkimin models [18] are very complex, due to multiple scattering, their theoretical treatment is a laborious task. That led other authors to restrict the scattering analysis to a limited frequency range [5], [24] to [27]. Later, Hirsekorn [28] and [29], Stanke and Kino [30], and Weaver [31] developed general solutions valid for all grain size to wavelength ratios and for cubic symmetry polycrystalline materials. However, other researchers demonstrated that those general solutions failed in more complex microstructures, such as the commercial aluminium alloys [32] and two-phase sintered powders [33]. More recent works deal with advances related to the interaction of ultrasound waves with polycrystalline materials. Yang et al. [34] studied the shape effect of elongated grains on attenuation. Arguelles and Turner [35] evaluated the errors resulting from neglecting the grain size distribution and using an average grain size. Ryzy et al. [36] used a semi-analytical attenuation model that considers the grain morphology and incorporates an exact spatial two-point correlation function. The authors concluded that the grain shape has a strong effect on attenuation in the Rayleigh-stochastic transition region. Rokhlin et al. [37] presented a model that includes second-order multiple scattering, applicable to all frequency ranges, providing small relative errors on both longitudinal and transverse attenuations for low anisotropy. Sha [38] extended the second-order attenuation (SOA) model for elastic waves in texture-free materials to textured polycrystals with ellipsoidal grains of arbitrary crystal symmetry. The predicted attenuation results of this work agree well with the literature on a textured stainless steel polycrystal.

Numerical or grid-based methods are also powerful tools in the analysis of the propagation in scattering media. The improvement of computational resources makes possible the implementation of those methods to study increasingly complex interactions [39]. Recent works used finite element methods (FEM) to accurately mimic wave propagation through a rather complex material [40] to [44]. Norouzian, et al. [45] use DREAM.3D tool to construct material volumes with lognormal grain-size distributions. The results show that the correlation between attenuation and distribution width can be modelled with a power law, and the frequency dependence of attenuation has been shown to be strongly depend on the distribution width.

The main advantage of these methods, when compared to analytical approaches, is related to the fact that they allow microscopic scale analysis instead of an averaged effective medium. No assumptions have to be made concerning grain statistics, anisotropy degree, or multiple scattering events [36]. Limitations related to frequency range, model dimensions and grid discretization tend to be strongly reduced, in the future, with the increase of computational resources.

One technique widely established for the generation of polycrystalline material morphologies is the Voronoi tessellation [40] to [50] for 2-D or 3-D dimensions. Details about that approach can be found in [50]. The major drawback of elastic wave models based on low-order finite difference or finite element schemes is the large number of grid points per wavelength required to avoid numerical dispersion. The k-Wave toolbox for Matlab can be used as an alternative to those approaches. It uses a Fourier domain pseudospectral method for the simulation and reconstruction of photoacoustic wave fields in a faster way. Also, it uses less memory and is user friendly [51] and [52]. Fewer spatial and temporal grid points are needed for accurate simulations [51]. Recently many authors have used the k-Wave toolbox in applications related to attenuation in ultrasonic computed tomography [53], guided waves in layered structures [54] and [55] time domain, power law attenuation in breast and liver tissues [56], one-sided ultrasonic nondestructive evaluation [57], high intensity focused ultrasound [58], nonlinear ultrasound propagation in absorbing media [59], ultrasonic transducers field modelling [60], and 3-D ultrasound imaging [61].

In the present work, the authors propose a simulation model to evaluate the scattering attenuation in structures with complex scatterer distributions. It uses the experimental attenuation knowledge in nodular cast iron to infer the scatterer size and its power dependence, as well as the concentration that best mimics the structure behaviour. The $\mathrm{k}$-Wave toolbox is used to construct the simulator. Additionally, the results provided by the theoretical and simulation models are presented and a discussion is also made about the feasibility of the Truell et al. [62] and Papadakis [22] models for attenuation evaluation in nodular cast iron.

\section{ULTRASONIC SCATTERING ATTENUATION}

\subsection{Theory}

For the Rayleigh domain, characterized by a wavelength much higher than the scatterer size, Ying and Truell [27] presented a model for the total scattering cross-section, assuming the scatterers are spherical, solid, elastic, isotropic, have uniform size 
and are embedded in a solid elastic matrix. Based on those assumptions, Truell et al. [62] presented an expression for the ultrasonic scattering attenuation evaluation of longitudinal waves:

$$
\alpha_{s T}=\frac{2 \pi}{9} N g \frac{\omega^{4}}{v_{l}{ }^{4}} r^{6},
$$

where $N$ is the number of scatterers per unit volume, $\omega$ is the angular frequency, $v_{l}$ represents the longitudinal propagation velocity, $r$ is the scatterer radius, and $g$ denotes a factor related to the elastic properties of the medium. The original $g$ expression in [27] can be changed to encompass the longitudinal $\left(v_{l}\right)$ and transversal $\left(v_{t}\right)$ velocities:

$$
\begin{aligned}
& g=\left(\frac{3\left(\frac{v_{l 1}}{v_{t 1}}\right)^{2}}{\left(3\left(\frac{v_{l 2}}{v_{t 2}}\right)^{2}-4\right) \frac{\mu_{2}}{\mu_{1}}+4}-1\right)^{2}+ \\
& +\frac{1}{3}\left(1+2\left(\frac{v_{l 1}}{v_{t 1}}\right)^{3}\right)\left(\frac{v_{t 1} \mu_{2}}{v_{t 2} \mu_{1}}-1\right)^{2}+ \\
& +40\left(2+3\left(\frac{v_{l 1}}{v_{t 1}}\right)^{5}\right) \text {. } \\
& \left(\frac{\frac{\mu_{2}}{\mu_{1}}-1}{2\left(3\left(\frac{v_{l 1}}{v_{t 1}}\right)^{2}+2\right) \frac{\mu_{2}}{\mu_{1}}+\left(9\left(\frac{v_{l 1}}{v_{t 1}}\right)^{2}-4\right)}\right)^{2},
\end{aligned}
$$

where $\mu$ is the shear modulus given by $v_{t}^{2} \rho ; \rho$ is the density, and the indexes 1 and 2 are related to the surrounding medium and scatterer, respectively.

Based on a similar work developed by Bathia and Moore [63], Papadakis [6] and [22] introduced a correction factor for the attenuation considering the effect of the grain size distribution. The author also showed a way of obtaining the correction factor in solids using two-dimension micrographs. The scatterer volume correction factor $(T)$ is:

$$
T=\frac{4 \pi}{3} \frac{\overline{r_{n}{ }^{6}}}{\overline{r_{n}^{3}}},
$$

where $\overline{r_{n}{ }^{6}}$ and $\overline{r_{n}^{3}}$ are the average scatterer radius values of the sixth and third power, respectively, obtained from micrograph samples. From Eq. (3), it is clear that the scattering effects of a single large scatterer are much greater than the effect summation of many small scatterers having the same volume. Taking the correction factor into account, the scattering attenuation is given as [6]:

$$
\begin{aligned}
\alpha_{s P}= & N T^{2} \frac{4 \pi^{3} f^{4}}{v_{l}^{4}} . \\
& \cdot\left(\left(\frac{1}{3} \frac{\Delta \rho^{2}}{\rho_{1}^{2}}+\frac{\Delta L^{2}}{L_{1}^{2}}-\frac{8}{3} \frac{\Delta L \Delta \mu}{L_{1}^{2}}+\frac{32}{15} \frac{\Delta \mu^{2}}{L_{1}^{2}}\right)+\right. \\
& \left.+\left(\frac{L_{1}}{\mu_{1}}\right)^{3 / 2}\left(\frac{2}{3} \frac{\Delta \rho^{2}}{\rho_{1}^{2}}+\frac{8}{15}\left(\frac{L_{1}}{\mu_{1}}\right) \frac{\Delta \mu^{2}}{L_{1}^{2}}\right)\right),
\end{aligned}
$$

where $f$ is the frequency, $L_{1}$ and $\mu_{1}$ are the longitudinal and the shear modulus of surrounding medium, respectively, and $\Delta \rho, \Delta L, \Delta \mu$ are the differences in density, longitudinal modulus, and shear modulus between scatterers and the surrounding medium, respectively. Although Eq. (4) uses a different approach for the attenuation calculation, the results are similar to those provided by the Truell model for uniform scatterer sizes.

\subsection{Experimental Attenuation}

The attenuation is the result of acoustic wave interactions with the propagation medium. Experimentally, the attenuation can be calculated by collecting the front surface reflection and two back wall ultrasound pulses from samples with parallel faces, as illustrated in Fig. 1. Following that approach, the authors studied different methodologies to calculate the ultrasonic attenuation in nodular cast iron samples [64], and concluded that Eq. (5) provided more reliable results, because it does not take into account the reflection coefficient between water and sample, which is frequency-dependent, as demonstrated experimentally [64]:

$$
\alpha=\frac{1}{2 L} \ln \left(\frac{A_{1} A_{s} D_{c}}{A_{1}^{2}+A_{2} A_{s}}\right) .
$$

In Eq. (5), $L$ is the sample thickness, $A_{1}$ and $A_{2}$ are the first and second back wall signals from samples, $A_{s}$ is the reflected signal on the sample front face, and $D_{c}$ is the diffraction correction coefficient for the sample path. The attenuation $(\alpha)$ unit is $[\mathrm{Np} / \mathrm{m}]$, where $\mathrm{Np}$ is the symbol of neper, which is a logarithmic unit for ratios of measurements of physical quantities. The neper and $\mathrm{dB}$ are related by the following relationship: $1 \mathrm{~Np}=8.686 \mathrm{~dB}$ 


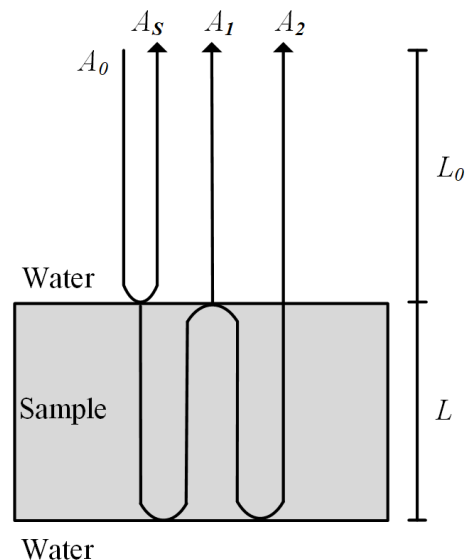

Fig. 1. Setup for attenuation measurement

\section{3 k-Wave Simulation}

The simulation of elastic wave propagation has many applications in ultrasonic non-destructive testing. The open-source k-Wave toolbox is an easy-to-use time-domain forward model based on a k-space pseudospectral time-domain solution to couple first-order acoustic equations for homogeneous and heterogeneous media in one, two, and three dimensions [51]. That tool makes possible the modelling of arbitrary sources, detecting surfaces with directional elements, and wave propagation that can account for nonlinearity, acoustic heterogeneities and power-law absorption. It is also possible to use optional input parameters to adjust the visualization and performance, including making wave propagation movies for presentations and running simulations on graphics processing units (GPU). The available geometry creation functions allow both Cartesian and grid-based geometries, such as circles, arcs, disks, spheres, shells or balls. As an example, Fig. 2 shows a three-dimensional model for a circular transducer (top) radiating into a sample with randomly distributed scatterers. The main drawback of $\mathrm{k}$-Wave in ultrasonic non-destructive testing is related to the existence of large contrasts between media. Fourier-based methods such as k-Wave are effective when everything is smooth, and the contrasts are not too big (the code was originally designed for modelling ultrasound in biological tissue, where the contrasts are low). For large contrasts, oscillatory errors can be accumulated, because the steep edges cannot be represented very well by a small number of Fourier components. Some caution must be taken in such situations. Smoothing the density and propagation velocity or keeping the time step small will help to reduce that effect.

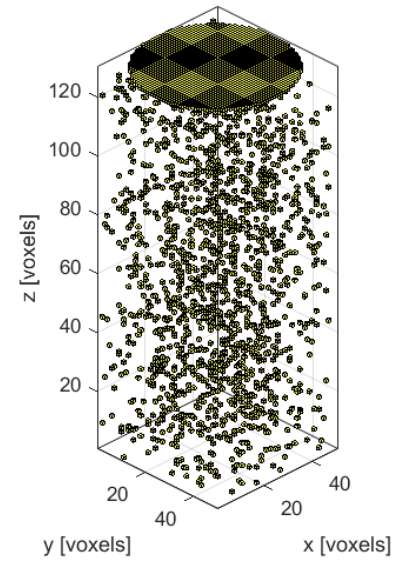

Fig. 2. Three-dimensional simulation model

\section{RESULTS AND DISCUSSION}

\subsection{Samples Granulometry Analysis}

Six machined cast iron samples with parallel surfaces within $15 \mu \mathrm{m}$ and $13.1 \pm 0.05 \mathrm{~mm}$ in thickness were used in this study (Fig. 3). The samples were prepared using standard metallographic methods. First, they were mechanically polished using metallographic carbon silicate sandpaper with decreasing granulometry (P180, P1000, and P2500) and a final polishing by a $3-\mu \mathrm{m}$ diamond suspension. The microstructure of the processed zones was examined using optical microscopy, with a $200 \times$ magnification.

The typical microstructure of such samples is shown in Fig. 4, where the precipitated graphite nodules (in black) embedded in a ferrite/perlite matrix are easily identified, as are the grain boundaries. It was observed in all samples that the nodules present non-uniform shapes and have a large wide range of sizes that can vary from less than one micrometre to several tens of micrometres (the larger measured radius was $35 \mu \mathrm{m})$.

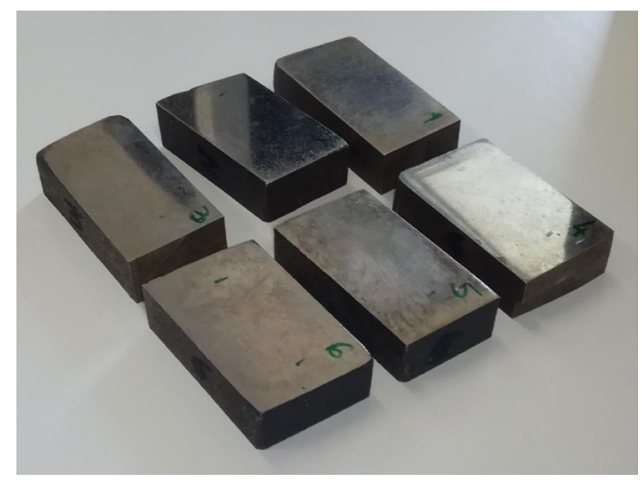

Fig. 3. Cast iron samples 


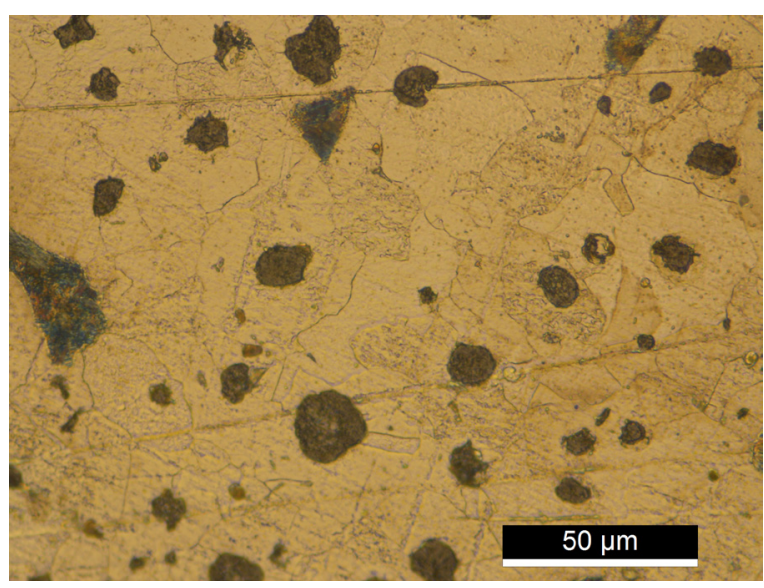

Fig. 4. Optical micrograph of a nodular cast iron sample

Ten micrograph images were taken from each sample, corresponding to an analysis section of $10.32 \mathrm{~mm}^{2}$. The ImageJ free package software was used to determine the nodules' size [65], which were considered to be spherical. The nodule size distribution is presented in Fig. 5. Most of the nodules have a radius less than $10 \mu \mathrm{m}$. The spatial variability of the nodules' size in different regions of each sample is low. The standard deviation of the nodule size divided by its average value obtained for all images of each sample was about $8.9 \%$. Also, for each sample, the total number of nodules was used to obtain the lognormal distribution parameters. The mean and standard deviation are presented in Table 1.

\subsection{Experimental Attenuation Evaluation Setup and Results}

The experimental immersion setup for the attenuation evaluation is presented in Fig. 6. A pulser/receiver is used to excite a broadband $8 \mathrm{MHz}$ central frequency transducer and to collect, amplify, and filter the reflected signals. Then, the acquired signals are displayed in an oscilloscope and transferred to a computer for further processing. The transducer is moved using a computer-controlled micro-positioning system.

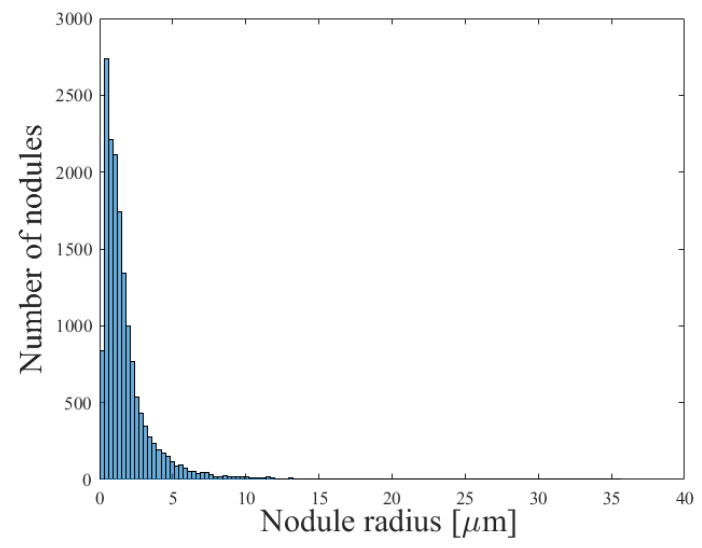

Fig. 5. Samples' nodule size distribution

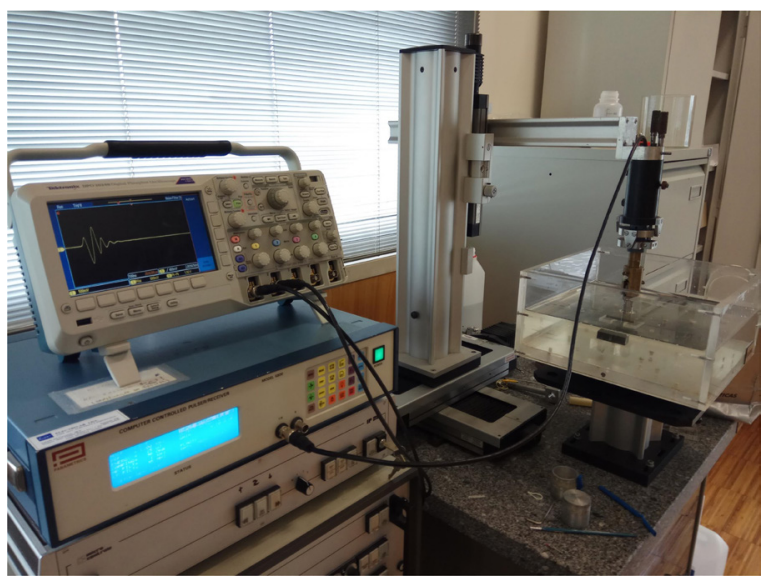

Fig. 6. Experimental setup for attenuation evaluation

All samples characterized in section 2.1 were used in the study. Four acquisitions were accomplished in different regions of each sample to take into account their structural variation. Then, using Eq. (5) an average attenuation of $11.96 \mathrm{~Np} / \mathrm{m}$ was obtained,

Table 1. Lognormal fitting parameters for each sample

\begin{tabular}{lcccccc}
\hline Sample & 1 & 2 & 3 & 4 & 5 & 6 \\
\hline Mean $[\mu \mathrm{m}]$ & 0.2335 & 0.1178 & 0.4310 & 0.1007 & 0.0671 & 0.2420 \\
\hline Standard deviation $[\mu \mathrm{m}]$ & 0.9492 & 0.8026 & 0.7862 & 0.8751 & 0.8545 & 0.9161 \\
\hline
\end{tabular}

Table 2. Acoustic properties of cast iron components

\begin{tabular}{lccccc}
\hline & $\begin{array}{c}\text { Longitudinal velocity } \\
{[\mathrm{m} / \mathrm{s}]}\end{array}$ & $\begin{array}{c}\text { Transversal velocity } \\
{[\mathrm{m} / \mathrm{s}]}\end{array}$ & $\begin{array}{c}\text { Longitudinal modulus } \\
{[\mathrm{GPa}]}\end{array}$ & $\begin{array}{c}\text { Shear modulus } \\
{[\mathrm{GPa}]}\end{array}$ & $\begin{array}{c}\text { Density } \\
{\left[\mathrm{kg} / \mathrm{m}^{3}\right]}\end{array}$ \\
\hline Pearlite/Ferrite & 5830 & 3090 & 269 & 75.6 & 7920 \\
\hline Graphite & 4210 & 2030 & 38 & 8.9 & 2170 \\
\hline
\end{tabular}


with a standard deviation (SD) of $1.22 \mathrm{~Np} / \mathrm{m}$ and root mean square error (RMSE) of $1.10 \mathrm{~Np} / \mathrm{m}$ for the 24 measurements.

\subsection{Attenuation Evaluation Using Analytical Models}

In order to evaluate the scattering attenuation in the cast iron samples, important parameters as longitudinal and shear velocities, longitudinal and shear moduli, and density related to the matrix (pearlite/ferrite) and scatterers (graphite), must be known. They are represented in Table 2 [24].

The scatterer concentration knowledge is also required to estimate the attenuation. Thus, the twodimensional scatterer concentration is obtained relating the total amount of nodules $\left(n_{T}\right)$ with the area of micrograph $\left(A_{T}\right)$, as follows:

$$
N_{A}=\frac{n_{T}}{A_{T}} .
$$

Then, the scatterer concentration $(N)$ can be calculated using the following equation [66]:

$$
N=\left(\frac{\pi}{6 f_{g}}\right)^{\frac{1}{2}}\left(\alpha_{N} N_{A}\right)^{\frac{3}{2}},
$$

where $f_{g}$ is the graphite nodule fraction that is about $3.33 \%$ for the analysed images, and $\alpha_{N}=1.25$, which is a parameter related to the width of the nodule size distribution.

To infer the real scatterer effect in the attenuation, the scatterers that contribute to the attenuation should be determined. To do so both the Truell and Papadakis models will be used and their performance analysed.

Due to the wide nodular distribution observed in the samples, the Truell model given by Eq. (1) cannot be used to calculate the scattering attenuation, because it uses the same size scatterers. Thus, in order to encompass all scatterer sizes found in the samples, the authors present a new equation for the attenuation, based on Eq. (1), which takes into account the summation of the different classes of scattering nodules:

$$
\alpha_{s T m}=\sum_{i=1}^{n} \frac{2 \pi}{9} g \frac{\omega^{4}}{v_{l}^{4}} N_{i} r_{i}^{6} .
$$

In Eq. (8), $n$ is the total number of scatterer classes, $r_{i}$ and $N_{i}$ are the average radius and scatterer concentration of each class, respectively. Thirty-five scatterer classes with $1 \mu \mathrm{m}$ step were considered for the attenuation calculation. The scatterer concentration of each class is a fraction of the one obtained by Eq.
(7). From Eq. (8), resulted an attenuation of 2.67 $\mathrm{Np} / \mathrm{m}$.

The different attenuation values provided by the Truell model and experimental approach could be due to the fact that the Truell model makes use of spherical, solid, elastic, and isotropic scatterers. In addition, the referred model assumes that there is a sharp variation of the properties between the scatterers and the surrounding media. However, the nodular cast iron structure is rather more complex, especially its matrix, which is usually formed by ferrite around the nodules, with a homogeneous structure, and by pearlite in other regions with a lath-type structure with some degree of heterogeneity [24]. Therefore, the boundary effect between scatterers and matrix is certainly more complex than the one presented in the model.

The Papadakis model takes into account the scatterer size distribution, which is an improved version of the Truell theory. Generally, in practical materials, such as cast iron, the scatterers are not all the same size and follow a distribution that can be evaluated. The correction factor (see Eq. (3)), requires the knowledge of $\overline{r_{n}^{6}}$ and $\overline{r_{n}^{3}}$ :

$$
\begin{aligned}
& \overline{r_{n}^{6}}=\sum_{i=1}^{n} \frac{r_{i}^{6}}{n}, \\
& \overline{r_{n}^{3}}=\sum_{i=1}^{n} \frac{r_{i}^{3}}{n} .
\end{aligned}
$$

Using the experimental scatterer distribution (see Fig. 5), resulted $\overline{r_{n}{ }^{6}}=3.5 \times 10^{5} \mu \mathrm{m}^{6}$ and $\overline{r_{n}^{3}}=54.73 \mu \mathrm{m}^{3}$ for the whole sample area analysed, and $T=2.68 \times 10^{-14} \mathrm{~m}^{3}$.

Then, using Eq. (4), where the right side is essentially composed by the physical parameters of matrix and scatterers presented in Table 1, resulted $\alpha_{s P}=370 \mathrm{~Np} / \mathrm{m}$, which is very large compared to the experimental attenuation. In addition to the explanation made for the Truell model deviation in relation to the experimental attenuation, which also applies to the Papadakis model, other important factors can contribute to the observed discrepancy between theory and experiment. Perhaps, the most important one deals with the scatterer distribution obtained from the micrograph images. In the Papadakis model, all scatterer sizes and shapes were taken into account for the attenuation calculation that originates a very high scatterer concentration value. However, the authors concluded the attenuation decreases sharply if the lower size scatterers are discarded, as illustrated in Fig. 7. That tendency is expected because the 
scatterer concentration $(N)$ falls more quickly than the correction factor $(T)$. For instance, if all scatterers lower than $7 \mu \mathrm{m}$ are discarded, the attenuation is 15 $\mathrm{Np} / \mathrm{m}$, which is very close to the experimental one.

Finally, the Papadakis model also presents limitations in predicting the scattering attenuation in the cast iron samples.

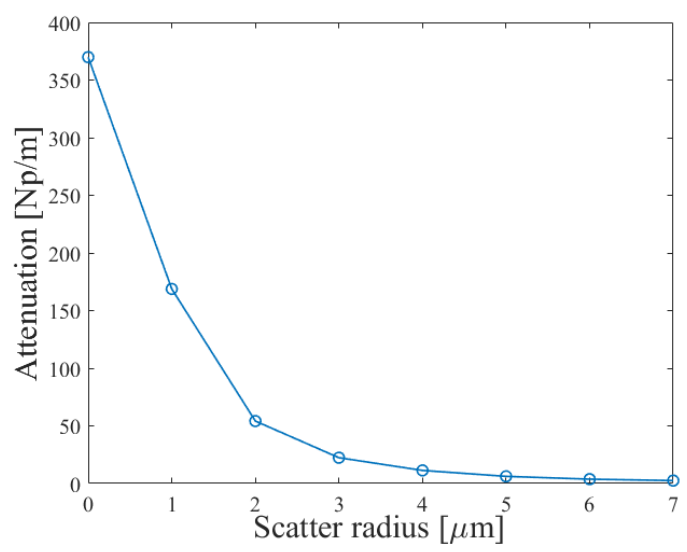

Fig. 7. Attenuation behaviour versus scatterer size

\subsection{Simulation Model}

The simulation of the cast iron nodular structure shown in Fig. 4 is difficult due to the non-uniformity of scatterers in size and shape. Here, the authors propose a simplified model assuming all scatterers with the same size and known concentration that leads to the experimental attenuation value. For that purpose, it is assumed that the attenuation is directly proportional to the scatterer concentration (see Eq. (11)), which is in accordance with the theoretical models [21] and [60] and is a common-sense assumption. The equivalent scatterer size $\left(r_{e q}\right)$ and its exponent dependence $(n)$ will be determined based on the inverse problem, using the experimental attenuation. The simplified attenuation expression based on the previous assumptions is as follows,

$$
\alpha=C N\left(r_{e q}\right)^{n},
$$

where $C$ and $n$ are constants to be determined. Eq. (11) follows the Rayleigh scattering model, and only the geometric parameters are of interest, such as the scatterer concentration, equivalent scatterer size, and its exponent dependence. The frequency dependence and the matrix and scatterer properties are included in the constant $C$, because the study related to the attenuation variation with these parameters is outside the scope of this work.
Using k-Wave, a simulation model with dimensions $x=6 \mathrm{~mm}, y=6 \mathrm{~mm}$ and $z=40 \mathrm{~mm}$, similar to the one illustrated in Fig. 2, was defined by a computational grid for simulation purposes. For the scattering attenuation evaluation, an ultrasonic probe is located at the bottom of the model $(z=0)$, working as a transmitter and another at $z=30 \mathrm{~mm}$ working as a receiver. Two signals are collected at the receiver: a reference signal $a_{0}$ (model without scatterers) and a signal $a_{s}$ considering the model filled up with randomly distributed scatterers. The scattering attenuation is then given by:

$$
\alpha_{\text {sim }}=\frac{1}{d} \ln \left(\frac{a_{0}}{a_{s}}\right),
$$

where $d=30 \mathrm{~mm}$ is the distance between the probes. The two probes are $6 \mathrm{~mm}$ in diameter, like the one used in the experimental measurements (see section 2.2). The probe excitation was made by a tone burst, as shown in Fig. 8, to mimic the experimental transducer response illustrated in Fig. 9.

The first set of simulations were carried out for scatterers of $r_{e q}=60 \mu \mathrm{m}$, corresponding to a grid point spacing of $120 \mu \mathrm{m}$ (cuboid edge). The scatterer concentration was varied from $0 \mathrm{~mm}^{-3}$ to $100 \mathrm{~mm}^{-3}$ with a step of $10 \mathrm{~mm}^{-3}$, and ten signals $a_{s}$ were collected for each concentration value. The corresponding attenuation was then calculated using Eq. (12), and the results are shown in Fig. 10. An average attenuation value for each concentration was considered. As expected, the attenuation increases as $N$ also increases. That behaviour is more pronounced for lower concentrations. In the boxplot of the simulated data (Fig. 10) the central mark is the median, the edges of the box are the $25^{\text {th }}$ and $75^{\text {th }}$ percentiles, the whiskers extend to the most extreme data points (not considering the outliers, which are plotted individually as crosses).

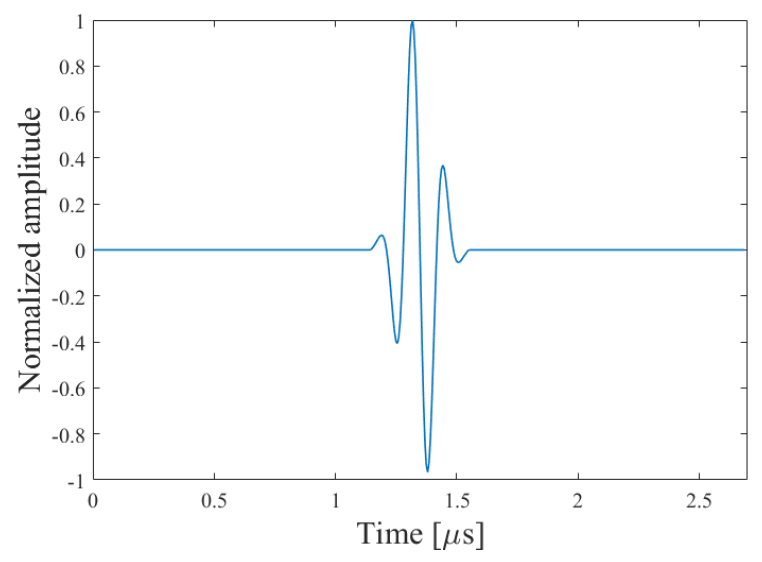

Fig. 8. Simulated tone burst 


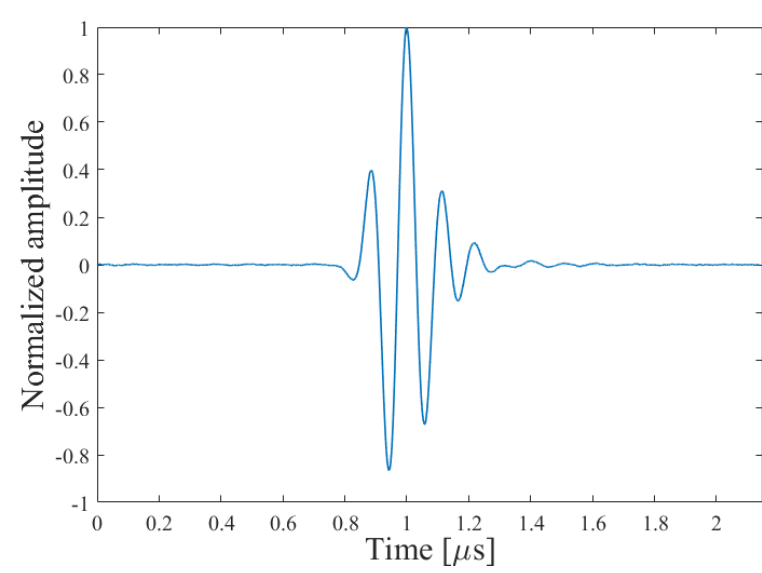

Fig. 9. Experimental transducer response

For the simulated scatterer size, the concentration value that leads to the experimental attenuation calculated in section $2.2\left(\alpha_{\exp }=11.96 \mathrm{~Np} / \mathrm{m}\right)$, is about $3.2 \mathrm{~mm}^{-3}$. This result is more clearly observed in Fig. 11, which corresponds to an expansion of Fig. 10.

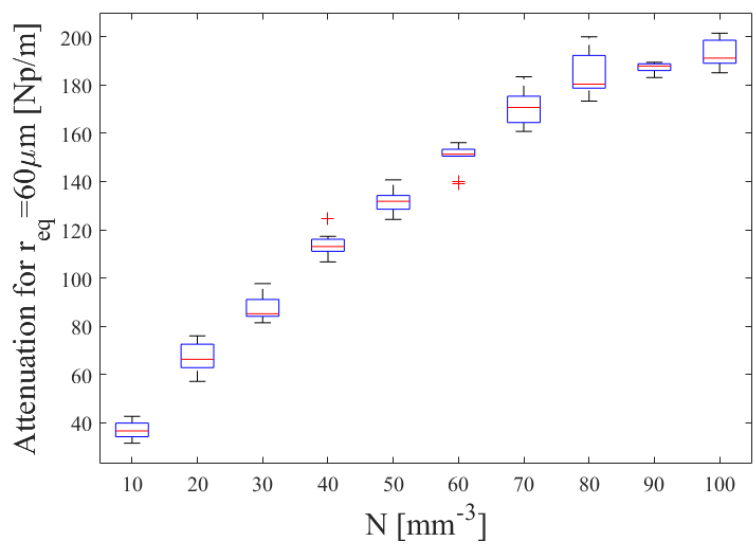

Fig. 10. Simulated attenuation for $r_{e q}=60 \mu \mathrm{m}$ versus scatterer concentration

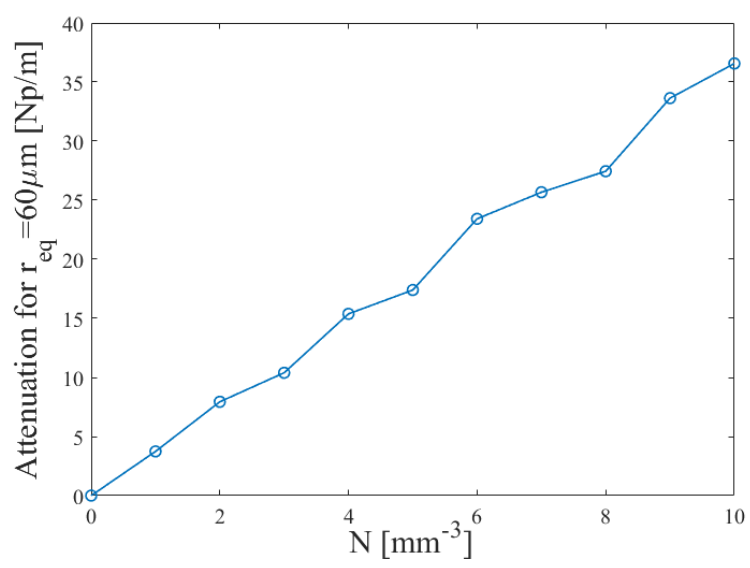

Fig. 11. Zoomed version of Fig. 10 for lower concentrations
In order to establish a correlation between the scatterer concentration $(N)$ and size $\left(r_{e q}\right)$, additional attenuation simulations were carried out using different scatterer sizes, resulting in the concentrations (presented in Table 2) that lead to the experimental attenuation. A power fitting applied to the values in Table 2 gave rise to Eq. (13), whose behaviour is illustrated in Fig. 12, where a remarkable goodness of fit with $R^{2}=0.99$ is observed:

$$
N=4.527 \times 10^{-13}\left(r_{e q}\right)^{-5.173} .
$$

Table 2. Scatterers dimensions and concentration for $\alpha_{\text {exp }}=11.96 \mathrm{~Np} / \mathrm{m}$

\begin{tabular}{lccccc}
\hline$r_{e q}[\mu \mathrm{m}]$ & 40 & 50 & 60 & 70 & 80 \\
\hline$N\left[\mathrm{~mm}^{-3}\right]$ & 25.5 & 8.0 & 3.2 & 1.4 & 0.68 \\
\hline
\end{tabular}

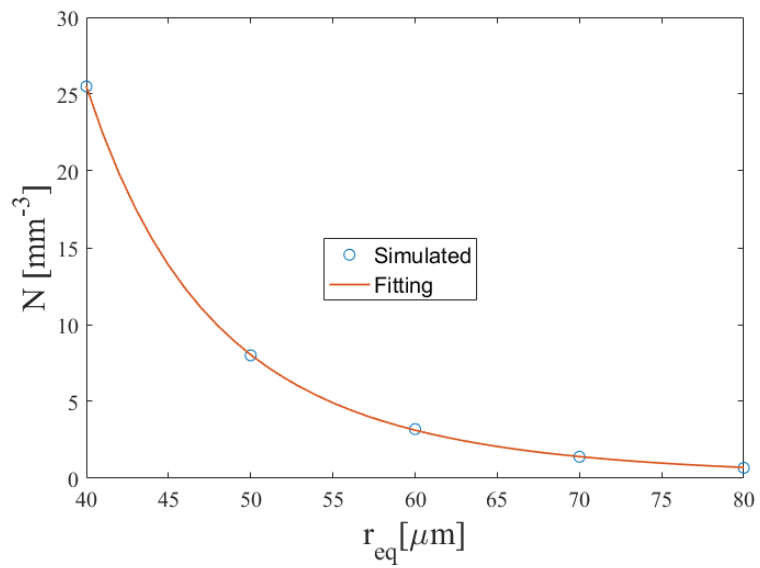

Fig. 12. Power fitting of scatterer concentration $N$ as function of $r_{e q}$

Solving the proposed simplified model given by Eq. (11), in order to extract the scatterer concentration:

$$
N=\frac{11.96}{C}\left(r_{e q}\right)^{-n}
$$

and comparing the Eqs. (13) and (14), results $C=2.65 \times 10^{13}$ and $n=5.173$, which allows writing Eq. (11) as:

$$
\alpha=2.65 \times 10^{13} N\left(r_{e q}\right)^{5.173},
$$

where the attenuation $[\mathrm{Np} / \mathrm{m}]$ is a function of the fifth power of the scatterer size, which is not far from the trend of Eq. (1), as expected. It should be pointed out that Eq. (15) resulted from the studied cast iron samples illustrated in Fig. 4, whose attenuation was experimentally calculated by Eq. (5). Thus, based on the previous knowledge of experimental 
attenuation, simulation models can be created varying the parameters $N$ and $r_{e q}$, according to Eq. (14). This allows mimicking structures with complex scatterer distributions in terms of uniform scatterer concentration and size.

The presented simulation model considers the geometrical factors (size and concentration of scatterers) and can be used to mimic the microstructure of cast iron for a specific excitation frequency. The scatterer equivalent size can be defined a priori and the concentration calculated by Eq. (14) to establish the simulation model.

\section{CONCLUSIONS}

This work aimed to evaluate the ultrasonic scattering attenuation of structures with complex scatterer distributions. The idea was to predict the attenuation behaviour for such complex structures by developing a simulation model based on the experimental attenuation results. That was motivated by the limitations presented by the Truell and Papadakis theoretical models that provide ultrasonic scattering attenuation results quite inconsistently when compared with the experimental one. That conclusion was observed for nodular cast-iron structures, and the authors believe that the same results are expected for materials having inhomogeneous scatterer sizes and concentrations. The $\mathrm{k}$-Wave simulation model developed in this work allows an easy and customized implementation, where the scatterer size and concentration can be varied to mimic the experimental ultrasonic scattering attenuation measured in inhomogeneous structures.

\section{ACKNOWLEDGEMENTS}

This research is sponsored by FEDER funds through the program COMPETE - Programa Operacional Factores de Competitividade - and by national funds through FCT - Fundação para a Ciência e a Tecnologia - under the project UIDB/00285/2020.

\section{REFERENCES}

[1] Gür, C., Aydinmakina, B. (2010). Microstructural Characterisation of Ductile Irons by Measuring Velocity and Apparent Attenuation of Ultrasonic Waves. Insight, vol. 43, no. 11, p. 731-734.

[2] Prakash, P., Mytri, V.D., Hiremath, P.S. (2011). Digital microstructure analysis system for testing and quantifying the ductile cast iron. International Journal of Computer Applications, vol. 19, no. 3, p. 22-27, D0l:10.5120/23423062.
[3] Cheng, T.-W., Lui, T.-S., Chen, L.-H. (2012). Microstructural features and erosion wear resistance of friction stir surface hardened spheroidal graphite cast iron. Materials Transactions, vol. 53, no. 1, p. 167-172, D0l:10.2320/ matertrans.M2011224.

[4] Ristic, V.M. (1983). Principles of Acoustic Devices. John Wiley \& Sons, New York.

[5] Papadakis, E.P. (1965). Ultrasonic attenuation caused by scattering in polycrystalline metals. Journal of Acoustical Society of America, vol. 37, no. 4, p. 711-717, DOI:10.1121/1.1909401.

[6] Papadakis, E.P. (1981). Ultrasonic attenuation caused by Rayleigh scattering by graphite nodules in nodular cast iron. Journal of Acoustical Society of America, vol. 70, no. 3, p. 782787, D0I:10.1121/1.386916.

[7] Kruger, S.E., Rebello, J.M.A., Charlier, J. (2004). Broadband ultrasonic backscattering applied to nondestructive characterization of materials. IEEE Transaction on Ultrasonic and Frequency Control, vol. 51, no. 7, p. 832-838, DOI:10.1109/TUFFC.2004.1320742.

[8] Palanichamy, P., Joseph, A., Jayakumar, T., Baldev, R. (1995). Ultrasonic velocity measurements for estimation of grain size in austenitic stainless steel. NDT\&E International, vol. 28, no. 3, p. 179-185, D0l:10.1016/0963-8695(95)00011-L.

[9] Zeng, F., Agnew, S.R., Raeisinia, B., Myneni, G.R. (2010). Ultrasonic attenuation due to grain boundary scattering in pure niobium. Journal of Nondestructive Evaluation, vol. 29, no. 2, p. 93-103, D0I:10.1007/s10921-010-0068-2.

[10] Thompson, R. (2002). Elastic-wave propagation in random polycrystals: Fundamentals and application to nondestructive evaluation. Fink, M., Kuperman, W.A., Montagner, J.P., Tourin, A. (eds.). Imaging of Complex Media with Acoustic and Seismic Waves. Topics in Applied Physics, Springer-Verlag, Berlin, Germany, vol. 84, p. 233-257, D0l:10.1007/3-540-44680-X_9.

[11] Thompson, R.B., Margetan, F.J., Haldipur, P., Yu, L., Li, A., Panetta, P., Wasan, H. (2008). Scattering of elastic waves in simple and complex polycrystals. Wave Motion, vol. 45, no. 5, p. 655-674, D0I:10.1016/j.wavemoti.2007.09.008.

[12] Pawlicki, A.D., O'Brien, W.Jr. (2013). Method for estimating total attenuation from a spatial map of attenuation slope for quantitative ultrasound imaging. Ultrasonic Imaging, vol. 35, no. 2, p. 162-172, Dol:10.1177/0161734613478695.

[13] Lobkis, O.I, Yang, L., Li, J., Rokhlin, S.I. (2012). Ultrasonic backscattering in polycrystals with elongated single phase and duplex microstructure. Ultrasonics, vol. 52, no. 6, p. 694-705, DOI:10.1016/j.ultras.2011.12.002.

[14] Lee, S.C., Suen, J.M. (1989). Ultrasonic nondestructive evaluation of matrix structures and nodularity in cast irons. Metallurgical Transactions A, no. 20, p. 2399-2407, DOI:10.1007/BF02666675.

[15] Liu, X., Takamori, S., Osawa, Y. (2007). Effect of matrix structure on ultrasonic attenuation of ductile cast iron. Journal of Materials Science, vol. 42, p. 179-184, D0l:10.1007/ s10853-006-0845-6.

[16] Kruger, S.E., Rebello, J.M.A,, Charlier, J. (2002). Measuring cast iron graphite size by ultrasonic attenuation. AIP Conference Proceedings, vol. 21, p. 1531-1537, D0l:10.1063/1.1472975.

[17] Rayleigh, L. (1929). Theory of Sound. McMillan, New York. 
[18] Mason, W.P, McSkimin, H.J. (1947). Attenuation and scattering of high frequency sound waves in metals and glasses. Journal of Acoustical Society of America, vol. 19, no. 3, p. 464-473, DOI:10.1121/1.1916504.

[19] Mason, W.P., McSkimin, H.J. (1948). Energy losses of sound waves in metals due to scattering and diffusion. Journal of Applied Physics, no. 19, p. 940-946, D0l:10.1063/1.1697900.

[20] Roth, W. (1948). Scattering of ultrasonic radiation in polycrystalline metals. Journal of Applied Physics, no. 19, no. 10, p. 901-910, D0l:10.1063/1.1697896.

[21] Lifshitz. I., Parkhomovskii, G. (1950) Theory of propagation of ultrasonic waves in polycrystals. Journal of Experimental and Theoretical Physics, vol. 20, p.175-182.

[22] Papadakis, E.P. (1961). Grain size distribution in metals and its influence on ultrasonic attenuation measurement. Journal of Acoustical Society of America, vol. 33, no. 11, p. 16161621, D0I:10.1121/1.1908517.

[23] Papadakis, E.P. (1964). From micrograph to grain-size distribution with ultrasonic applications. Journal of Applied Physics, vol. 35, no. 5, p. 1586-1594, Dol:10.1063/1.1713671.

[24] Papadakis, E.P. (1965). Revised grain-scattering formulas and tables. Journal of Acoustical Society of America, vol. 37, p. 703-710, DOI:10.1121/1.1909399.

[25] Papadakis, E.P. (1963). Rayleigh and Stochastic Scattering of Ultrasonic Waves in Steel. Journal of Applied Physics, vol. 34, no. 2, p. 265-269, DOI:10.1063/1.1702596.

[26] Waterman, P., Truell, R. (1961). Multiple scattering of waves. Journal of Mathematical Physics, vol. 2, no. 4, p. 512-537, DOI:10.1063/1.1703737.

[27] Ying, C.F., Truell, R. (1956). Scattering of a plane longitudinal wave by a spherical obstacle in an isotropically elastic solid. Journal of Applied Physics, vol. 27, no. 9, p. 1086-1097, DOI:10.1063/1.1722545.

[28] Hirsekorn, S. (1982). The scattering of ultrasonic waves by polycrystals. Journal of Acoustical Society of America, vol. 72, no. 4, p. 1021-1031, D0I:10.1121/1.389206.

[29] Hirsekorn, S. (1983). The scattering of ultrasonic waves by polycrystals. II. Shear waves. Journal of Acoustical Society of America, vol. 73, no.4, p. 1160-1163, Dol:10.1121/1.389206.

[30] Stanke, F.E, Kino, G.S. (1984). A unified theory for elastic wave propagation in polycrystalline materials. Journal of Acoustical Society of America, vol. 75, no. 3, p. 665-681, D0I:10.1121/1.390577.

[31] Weaver, R. (1990). Diffusivity of ultrasound in polycrystals. Journal of the Mechanics and Physics of Solids, vol. 38, no. 1, p. 55-86, DOl:10.1016/0022-5096(90)90021-U.

[32] Man, C., Paroni, R., Xiang, Y., Kenik, E.A. (2006). On the geometric autocorrelation function of polycrystalline materials. Journal of Computational and Applied Mathematics, vol. 160, no. 1-2, p. 200-210, D0l:10.1016/j.cam.2005.01.044.

[33] Liu, D., Turner, JA. (2008). Influence of spatial correlation function on attenuation of ultrasonic waves in two-phase materials. Journal of Acoustical Society of America, vol. 123, no. 5, p. 2570-2376, D0I:10.1121/1.2896757.

[34] Yang, L., Lobkis, O.I., Rokhlin, S.I. (2011). Shape effect of elongated grains on ultrasonic attenuation in polycrystalline materials. Ultrasonics, vol. 51, no. 6, p. 697-708, DOI:10.1016/J.ultras.2011.02.002.
[35] Arguelles, A.P., Turner, J.A. (2017). Ultrasonic attenuation of polycrystalline materials with a distribution of grain sizes. Journal of the Acoustical Society of America, vol. 141, no. 6, p. 4347-4353, DOI:10.1121/1.4984290.

[36] Ryzy, M., Grabec, T., Sedlák, P., Veres, I.A. (2018). Influence of grain morphology on ultrasonic wave attenuation in polycrystalline media with statistically equiaxed grains. Journal of the Acoustical Society of America, vol. 143, no. 1, p. 219-229, DOI:10.1121/1.5020785.

[37] Rokhlin, S.I., Li, J., Sha, G. (2015). Far-field scattering model for wave propagation in random media. Journal of the Acoustical Society of America, vol. 137, no. 5, p. 2655-2669, DOI:10.1121/1.4919333.

[38] Sha, G. (2020). Attenuation and phase velocity of elastic wave in textured polycrystals with ellipsoidal grains of arbitrary crystal symmetry. Acoustics, vol. 2, no. 1, p. 51-72, DOI:10.3390/acoustics2010005.

[39] Van Pamel, A., Sha, A., Rokhlin, S.I., Lowe, M.J.S. (2016). Finiteelement modelling of elastic wave propagation and scattering within heterogeneous media. Proceedings of the Royal Society A, vol. 473, no. 2197, p. 1-21, Dol:10.1098/rspa.2016.0738.

[40] Ghoshal, G., Turner, J.A. (2009). Numerical model of longitudinal wave scattering in polycrystals. IEEE Transactions on Ultrasonics, Ferroelectrics, and Frequency Control, vol. 56, no. 7, p. 1419-1428, D0l:10.1109/TUFFC.2009.1197.

[41] Lan, B., Lowe, M., Dunne, F.P.E. (2014). Experimental and computational studies of ultrasound wave propagation in hexagonal close-packed polycrystals for texture detection. Acta Materialia, vol. 63, p. 107-122, D0l:10.1016/j. actamat.2013.10.012.

[42] Van Pamel, A., Brett, C., Huthwaite, P., Lowe, M. (2015). Finite element modelling of elastic wave scattering within a polycrystalline material in two and three dimensions. Journal of the Acoustical Society of America, vol. 138, no. 4, p. 23262336, Dol:10.1121/1.4931445.

[43] Shahjahan, S., Rupin, F., Aubry, A., Chassignole, B., Fouquet, T., Derode, A. (2014). Comparison between experimental and 2-D numerical studies of multiple scattering in inconel600 8 by means of array probes. Ultrasonics, vol. 54 , no. 1, p 358 367, Dol:10.1016/j.ultras.2013.06.012.

[44] Chassignole, B., Duwig, V., Ploix, M.-A., Guy, P., El Guerjouma, R. (2009). Modelling the attenuation in the ATHENA finite elements code for the ultrasonic testing of austenitic stainless steel welds. Ultrasonics, vol. 49, no. 8, p. 653-658, D0l:10.1016/j.ultras.2009.04.001.

[45] Norouzian, M., Islam S., Turner. J.A. (2020). Influence of microstructural grain-size distribution on ultrasonic scattering. Ultrasonics, vol. 102, p. 1-7, D0l:10.1016/j. ultras.2019.106032.

[46] Voronoi, G. (1908). Nouvelles applications des paramètres continus à la théorie des formes quadratiques. Premier mémoire. Sur quelques propriétés des formes quadratiques positives parfaites. Journal für die Reine und Angewandte Mathematik, no. 133, p. 198-287, D0l:10.1515/ crll.1908.133.97.

[47] O'Rourke, J. (1988). Computational Geometry in C, Cambridge University Press, Cambridge. 
[48] Zhang, P., Balint, D., Lin, J. (2011). An integrated scheme for crystal plasticity analysis: virtual grain structure generation. Computational Materials Science, vol. 50, no. 10, p. 28542864, DOI:10.1016/j.commatsci.2011.04.041.

[49] Zhang, K., Holmedal, B., Hopperstad, O., Dumoulin, S., Gawad, J., Van Bael, A., Van Houtte, P. (2015). Multi-level modelling of mechanical anisotropy of commercial pure aluminium plate: crystal plasticity models, advanced yield functions and parameter identification. International Journal of Plasticity, vol. 66, p. 3-30, D0l:10.1016/j.ijplas.2014.02.003.

[50] Quey, R., Dawson, P.R., Barbe, F. (2011). Large-scale 3D random polycrystals for the finite element method: Generation, meshing and remeshing. Computer Methods in Applied Mechanics and Engineering, vol. 200, no. 17-20, p. 1729-1745, D0l:10.1016/j.cma.2011.01.002.

[51] Treeby, B.E., Cox, B.T. (2010). k-Wave: MATLAB toolbox for the simulation and reconstruction of photoacoustic wave-fields. Journal of Biomedical Optics, vol. 15, no. 2, p. 21314-121314-12, Dol:10.1117/1.3360308.

[52] Treeby, B., Jaros, J., Rendell, A., Cox, B. (2012). Modeling nonlinear ultrasound propagation in heterogeneous media with power law absorption using a k-space pseudospectral method. Journal of the Acoustical Society of America, vol. 131, no. 6, p. 4324-4336, D0l:10.1121/1.4712021.

[53] Pérez-Liva, M., Herraiz, J.L., Udías, J.M., Miller, E., Cox, B.T., Treeby, B.E. (2017). Time domain reconstruction of sound speed and attenuation in ultrasound computed tomography using full wave inversion. Journal of the Acoustical Society of America, vol. 141, no. 3, p. 1595-1604, DOI:10.1121/1.4976688.

[54] Brill, T.M., Klieber, C. (2016). Reflection and mode-conversion of ultrasonic leaky Lamb waves at inaccessible discontinuities in layered structures. 2016 IEEE International Ultrasonics Symposium, DOI:10.1109/ULTSYM.2016.7728587.

[55] Klieber, C., Brill, T.M. (2017). Mapping of ultrasonic Lambwave field in elastic layered structures using laser probes. Proceedings of Meetings on Acoustics, vol. 30, art. ID 065013, DOl:10.1121/2.0000603.

[56] Zhao, X., McGougha, R.J. (2018). Time-domain analysis of power law attenuation in space-fractional wave equations. Journal of the Acoustical Society of America, vol. 144, no.1, p. 467-477, DOI:10.1121/1.5047670.

[57] Almansourin, H., Venkatakrishnan, S., Bouman, C., SantosVillalobos, H. (2019). Model-based iterative reconstruction for one-sided ultrasonic nondestructive evaluation. IEEE Transactions on Computational Imaging, vol. 5, no. 1, p. 150164, DOI:10.1109/TCI.2018.2883218.

[58] Matsui, K., Azuma, T., Fujiwara, K., Takeuchi, H., Itani, K., Wang, J., Iwahashi, T., Kobayashi, E. Sakuma, I. (2017). Improving high-intensity focused ultrasound beam imaging via a backscattering suppression algorithm. Japanese Journal of Applied Physics, vol. 56, no. 5, p. 057301-1:057301-12, DOI:10.7567/JJAP.56.057301.

[59] Wang, K., Teoh, E., Jaros, J., Treeby, B. (2012). Modelling nonlinear ultrasound propagation in absorbing media using the k-Wave toolbox: experimental validation. 2012 IEEE International Ultrasonics Symposium, D0l:10.1109/ ULTSYM.2012.0130.

[60] Acquaticci, F., Guarracino, J.F., Gwirc, S.N., Lew, S.E. (2019). A polydimethylsiloxane-based axicon lens for focused ultrasonic brain stimulation techniques. Acoustical Science and Technology, vol. 40, no. 2, p. 116-126, D0l:10.1250/ ast.40.116.

[61] Cui, W., Qin, K. (2018). Fast 3-D ultrasonic imaging using time-domain synthetic aperture focusing techniques based on circular scan conversions. IEEE Transactions on Computational Imaging, vol. 4, no. 4, p. 632-639, DOI:10.1109/TCI.2018.2870303.

[62] Truell, R., Elbaum, C., Chick, B.B. (1969). Ultrasonic Methods in Solid State Physics, Academic Press, New Your, DOl:10.1016/C2013-0-12565-2.

[63] Bhatia, B.A. Moore, A.R. (1959). Scattering of HighFrequency Sound Waves in Polycrystalline Materials. Journal of Acoustical Society of America, vol. 31, p. 1140-1141, DOI:10.1121/1.1907843.

[64] Santos, M., Santos, J. (2019). Evaluation of three different approaches for the ultrasound attenuation coefficient measurement in nodular cast iron. Materials Evaluation, vol. 77, no. 5, p. 605-612.

[65] Schneider, C.A., Rasband, W.S., Eliceiri, K.W. (2012). NIH Image to ImageJ: 25 years of image analysis. Nature Methods, vol. 9, p. 671-675, D0l:10.1038/nmeth.2089.

[66] Pedersen, K.M., Tiedje, N.S. (2008). Graphite nodule count and size distribution in thin-walled ductile cast iron. Materials Characterization, vol. 59, no. 8, p. 1111-1121, D0l:10.1016/j. matchar.2007.09.001. 